Voix et Images

\title{
Le graffito urbain, forme brève du manifeste ou art mineur
}

\section{Jeanne Demers et Line Mc Murray}

Volume 15, numéro 2 (44), hiver 1990

Pratiques illicites

URI : https://id.erudit.org/iderudit/200835ar

DOI : https://doi.org/10.7202/200835ar

Aller au sommaire du numéro

Éditeur(s)

Université du Québec à Montréal

ISSN

0318-9201 (imprimé)

1705-933X (numérique)

Découvrir la revue

Citer cet article

Demers, J. \& Mc Murray, L. (1990). Le graffito urbain, forme brève du manifeste ou art mineur. Voix et Images, 15(2), 209-218. https://doi.org/10.7202/200835ar d'utilisation que vous pouvez consulter en ligne.

https://apropos.erudit.org/fr/usagers/politique-dutilisation/ 


\title{
Le graffito urbain, forme brève du manifeste ou art mineur*
}

\author{
par Jeanne Demers, Université de Montréal \\ et Line Mc Murray, chercheuse autonome, Montréal
}

$\int_{\text {(sing.) Lun ou l'autre sur la clôture de bois pourri, }}$ sur le vert glauque des portes de toilette (toilet).

Nicole Brossard 1

Every graffito can thus be seen and/or read as a miniature autobiography of a member of a society in the sense that the graffitist reveals a part of himself and his society in all that he writes.

Ernest L. Abel et Barbara E. Buckley ${ }^{2}$

La question du graffito, geste délinquant, à la limite du vandalisme, est scandaleuse par définition et l'on choque souvent à vouloir l'aborder. Mais comment s'en abstenir quand on sait que le phénomène connaît depuis au moins les années soixante une recrudescence évidente! Aucun mur européen ou nord-américain n'est à l'abri d'une éventuelle transcription, que celle-ci se fasse simple signature, qu'elle se prolonge en texte - monologue qui se transforme souvent en dia et multilogue - ou qu'elle privilégie la ligne, les formes, les pleins de toutes sortes. Et les visées sont rarement d'abord esthétiques. Ce que cherche la plupart du temps le graffiteur, c'est, par le biais d'un geste de transgression, d'exhiber devant un lecteur-graffitaire qu'il fantasme, ses idées politiques, sa conception du monde, ses rejets et ses choix, ses souffrances même et pourquoi pas, à l'occasion, sa joie.

Il nous aura fallu mener pendant plusieurs années des recherches sur le manifeste, littéraire et politique, pour que nous nous intéressions au phénomène du graffito. Ce dernier s'est imposé à nous au fur et à mesure que nous constations ses liens de parenté avec le texte manifestaire: même marginalité, même sens de

* La première partie de cet article résulte de la refonte de deux textes: une conférence faite par Jeanne Demers et Line Mc Murray lors du colloque 1987 des étudiants en urbanisme du Canada, à Montréal, et une intervention de Jeanne Demers à l'Université de Liège dans le cadre du programme des Arts et Sciences de la communication. Les photos sont de Josée Lambert.

1 Nicole Brossard, Sold-out, étreinte/illustration, Montréal, Éditions du Jour, 1973, p. 60.

2 Ernest L. Abel et Barbara E. Buckley, The Handwriting on the Wall, Westport (Conn.)/ Londres (G.-B.), Greenwood Press, 1977, p. 20. 
la contestation, même violence, même volonté de pouvoir sur l'autre, même goût de l'insulte, même "flirt" avec la récupération. Deux différences importantes toutefois: le type de support exigé par le graffito, soit le mur, la clôture, la vitrine plutôt que l'affiche, le dépliant, etc.; et son anonymat, si l'on met à part les cas d'autonomination, de pseudonymie ou encore les "œuvres" toujours signées d'artistes-graffiteurs. Mais autrement, quelle différence fondamentale y a-t-il, par exemple, entre le graffito montréalais - USINE DE DESTRUCTION DE LINDI$\mathrm{VIDU}^{3}$ - qui tire une partie de sa performativité de son lieu d'inscription, soit le mur d'une école, et ce passage du Refus global, texte de rupture s'il en fut:

Héritières de l'autorité papale, mécanique, sans réplique, grands maîtres des méthodes obscurantistes nos maisons d'enseignement ont dès lors les moyens d'organiser en monopole le règne de la mémoire exploiteuse, de la raison immobile, de lintention néfaste. ${ }^{4}$

Entre ces extraits d'un poème manifeste de Paul Chamberland

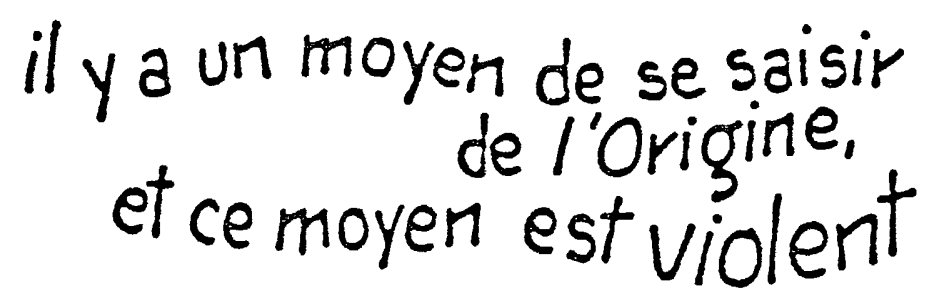

et ce commentaire ironico-philosophique illustré d'une pompe à essence et dont l's final est le signe du dollar:
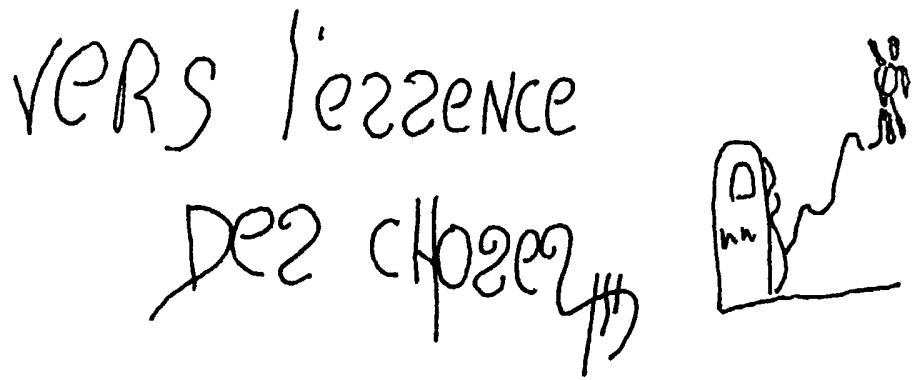

3 Jeanne Demers, Line McMurray et Josée Lambert, Montréal graffiti, Montréal, VLB éditeur, 1987 , p. 105. À moins d'indications contraires, tous les graffitis cités dans cet article sont tirés de ce livre et de Montréal graffiti bis, Montréal, VLB éditeur, 1988.

4 «Refus global „, Refus global, mimiographie originale déposée au Musée d'art contemporain de Montréal, p. 1-2.

5 Paul Chamberland, Demain les dieux naîtront, Montréal, IHexagone, 1974, p. 35.

6 Montréal graffiti, p. 20. 
Forme populaire d'un manifeste qui privilégierait la revendication individuelle plutôt que la collective, le graffito emprunte plusieurs visages en investissant l'espace public ouvert - la rue, les places, les ruelles, les cours - ou l'espace public fermé que constituent les stationnements souterrains, les tunnels, les salles des pas perdus, les toilettes, etc. Cet article qui emprunte son corpus à Montréal se limitera au graffito extérieur, celui qui s'étale au grand jour, à même la ville monumentale. Il ne sintéressera qu'indirectement à l'autre graffito, plus intime et plus inquiet qui se dissimule/dévoile dans les lieux clos. Mais ce n'est que partie remise...

\section{Bref aperçu historique et méthodologique}

À ce jour, les tentatives faites pour expliquer la recrudescence du graffito urbain ces trente dernières années sont rares. À peine peut-on citer celle de Richard Goldstein: le graffito se serait développé à New York, cité visuelle par excellence et qui, pour cette raison, attire les artistes graffitistes du monde entier ${ }^{7}$. Ce qui est sûr, c'est que le phénomène correspond à une tradition vieille comme la civilisation occidentale ${ }^{8}$. Un examen même rapide de la relativement pauvre bibliographie sur la question (une centaine de titres seulement d'ouvrages ou d'articles dont la majorité est en anglais) nous convainc en effet qu'il remonte au moins à l'Antiquité grecque et romaine. Et il débordait sans doute les strictes limites helléniques et latines puisque dans l'Ancien Testament, le livre de Daniel raconte l'histoire exemplaire, presque mythique, de Balthazar (Bel-Shar-Ousour), roi de Babylone, littéralement anéanti par les mots Mané, Thécel, Pharès qu'une main mystérieuse et prophétique avait inscrits sur un mur du palais, lors d'un banquet sacrilège. Il y avait de quoi: la main anonyme avait annoncé Mané ou Dieu a compté les jours de ton règne et il en a marqué la fin; Thécel: tu as été mis dans la balance, et tu as été trouvé trop léger; Pharès: ton royaume sera partagé 9 .

Rassurons-nous. Tous les graffiti ne peuvent prétendre à une telle performativité; encore que Plutarque relate dans ses Vies le cas de Tiberius Graccus qui aurait institué ses lois agraires en réponse aux réclamations du peuple romain inscrites un peu partout sur les colonnades, les murs des maisons, les monuments, en faveur d'une distribution aux pauvres des terres publiques ${ }^{10}$. Tous se situent toutefois au cœur même des préoccupations des sociétés qui les voient apparaître, quand ils ne relèvent pas, plus universellement, de pulsions fondamentales. C'est ainsi qu'en 67 après Jésus-Christ, la plèbe affamée de Pompéi multiplie les insultes et les reproches sur le socle des statues de Néron qui détourne au profit de

7 Hypothèse rapportée par Pascaline Cuvelier (Libération, 28 octobre 1981, p. 24) qui présente Richard Goldstein comme chroniqueur es graffiti.

8 Nous sommes d'accord avec Harvey D. Lomas à l'effet qu'il faille écarter la tentation, facile, de faire le rapprochement avec les inscriptions faites dans les cavernes par les hommes préhistoriques: It would appear that writing on walls before there were alternative modes of written communication has no connection with our subject [...]. « Graffiti: Some Observations and Speculations », The Psychoanalytic Review, vol. XL, no 1, printemps 1973, p. 73-74.

9 Cf. le Petit Larousse.

10 Cité par Allen Walker Read, Classic American Graffiti, Wisconsin, Maledicta Press, 1977, p. 21. 
ses plaisirs les richesses de l'Empire ${ }^{11}$. Et qu'à la même époque, les latrines de Rome sont tellement couvertes d'inscriptions obscènes - souvent en forme de poèmes, nous apprend Martial - que les autorités sévissent en y plaçant effigies de dieux ou emblèmes religieux quill deviendra scandaleux de maculer ou d'abîmer ${ }^{12}$.

Rien d'étonnant alors que les premiers à se pencher sur le phénomène aient été des historiens, des anthropologues, des folkloristes, le graffito étant reçu comme trace, artefact, document susceptible de fournir des précieuses informations sur un passé plus ou moins lointain, sur les gens, leurs comportements, les situations politiques, religieuses et autres auxquelles ils avaient à faire face, etc. Lintérêt des psychologues, vite passé du côté des sexologues et psychanalystes de tout acabit, est relativement récent, comme le signale Harvey $D$. Lomas qui explique le fait par le refoulé inévitable provoqué par le caractère scandaleux du graffito, surtout lorsque celui-ci se fait sexuel ou scatologique. Et il en veut pour preuve le refus de publication que son collègue Gershon Weltman et lui ont essuyé au début de leurs travaux ${ }^{13}$.

On l'aura déjà compris, un tel contexte ne favorisait guère les analyses originales, en particulier textuelles. Aussi les approches méthodologiques ontelles fini par se ressembler toutes, à quelques exceptions près, celle par exemple d'Allen Walker Read, Classic American Graffiti, dont la portée linguistique est indéniable ${ }^{14}$, et celle de David Ley et Roman Cybriwsky qui porte sur le graffito comme marqueur de territoire ${ }^{15}$. On pose à peu près les mêmes questions aux graffiti de la place du marché de l'Athènes antique, à ceux gravés dans la pierre aux XVe et XVIe siècles par les célèbres prisonniers de la tour de Londres ou plus tard incisés à même les verrières de certaines tavernes anglaises; aux très contemporains enfin, qu'ils surgissent sur les murs de nos villes, dans les toilettes de nos édifices publics ou sur les lieux touristiques les plus divers: en quoi les graffiti nous renseignent-ils sur le milieu qui leur a donné naissance? De quoi traitent-ils? À quels besoins répondent-ils? Peut-on enfin en dresser une typologie? Cela est tellement vrai que H.D. Lomas a trouvé légitime de présenter un tableau intitule Messages Then and Now dans lequel, sous une rubrique comme "Political Advertisement" ou "Sexual Solicitation ", il compare des graffiti du Pompéi de 79 après Jésus-Christ à d'autres relevés en 1965 à Los Angeles:

Hermes recommends
Calvetius as mayor

I am yours for 2 coppers
I

I
Edelman for Council

Marion \#25 16

À tort ou à raison, nous avons pensé que ces approches laissaient toujours ouverte la question, c'est-à-dire la définition même du graffito. Aussi, est-ce cette

11 M. Della Corte, «Grafiche Delta E. Di Maura-Cava De Tirveni «, Loves and Lovers of Ancient Pompëi, 1960, p. 24.

12 Cf. Ernest L. Abel et Barbara E. Buckley, op. cit., p. 5.

13 Harvey D. Lomas, op. cit., p. 71-72.

14 Allen Walker Read, op. cit.

15 David Ley et Roman Cybriwsky, «Urban Graffiti as Territorial Markers», Annals of the Association of American Geographer, vol. LXIV, 1974, p. 491-505.

16 Harvey D. Lomas, op. cit., p. 77. 
question qui constitue l'horizon de notre réflexion. Qu'est-ce que le graffito, audelà, bien entendu, de l'explication offerte par l'examen philologique du mot, d'origine italienne rappelons-le. Quelle sorte de texte est-il? Quel pacte installe-t-il entre le graffiteur et son lecteur, le graffitaire? Quel type de relations entretientil avec l'Institution, que celle-ci soit politique au sens étroit du terme, religieuse, linguistique, éducationnelle, etc. ? Quels liens établit-il avec lui-même en tant que microtexte partageant plus souvent qu'autrement un macrotexte, soit le mur sur lequel il est inscrit et auquel il collabore ${ }^{17}$ ? Toutes ces questions invitent à une approche pragmatique, approche qui s'intéresse d'abord à la dynamique de l'effet.

\section{Modes du graffito urbain: le cas Montréal}

Une telle approche accorde une grande importance à la réception du graffito, c'est-à-dire au point de vue du graffitaire. Encore faut-il pour y recourir, cerner d'abord les différents modes d'expression qu'il emprunte. Dans une grande ville comme Montréal, ces modes sont multiples. Ils couvrent toute la gamme des diverses fonctions que s'attribue de nos jours ce type d'inscription illégale: simple nomination, marque de territoire, protestation ou revendication, publicité pirate, déclaration d'intention, geste missionnaire, intervention ludique, etc.

Mais que faut-il entendre par "simple nomination", sinon la trace du passage, quelque part, de Pierre, Jean ou Jacques, maintenant comme il y a plusieurs siècles? Et sans autre projet que de sinscrire dans la mémoire du temps. La marque du territoire répond à un désir plus actuel, plus terrifiant et plus angoissant aussi: celui de clamer violemment son identité ou son appartenance à un groupe protecteur en délimitant une zone de risque pour le non-initié. Un bon exemple montréalais: les nombreux MASTER $B$ (pour maitre Bélanger, chef incontesté d'un gang de durs), concentrés à la station de métro HenriBourassa, mais que l'on trouve également ailleurs ${ }^{18}$.

Sans doute parce que très répandue, la fonction de protestation est la plus spontanément associée au graffito. Elle sert à rejeter une politique - BOYCOTTONS L'APPARTHEID ${ }^{19}$ ou U.S.A. ÉCCEURE PAS LE PEUPLE 20 -, une idéologie - le machisme (NON / $A U$ / $M A C H O^{21}$ ), par exemple, et ses avatars, la pornographie $(\overline{P O} Q)^{22}-$, un système d'éducation - BRAIN DAMAGE ${ }^{23}-$, etc. Son envers, la revendication, s'en prend aux sujets les plus divers et ainsi l'équation tourne souvent autour de thèmes essentiels tels l'espoir - IVRE $D^{\prime} E S P O I R^{24}$-, l'amour

17 Pour les notions de micro et de macrotexte: Jeanne Demers et Line McMurray, a Le privé est politique s, Atlantis, 1982, p. 27-35.

18 Montréal graffitis bis, p. 73.

19 Montréal graffiti, p. 116.

20 Ibid., p. 39.

21 Ibid., p. 29.

22 Ibid., p. 69.

23 Ibid., p. 102.

24 Ibid., p. 73. Notons que le $O$ d'ESPOIR ressemble étrangement à un $A$, connotant ainsi paradoxalement le mot anglais DESPAIR. 


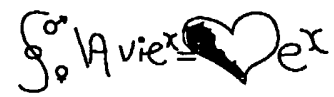

25

le bonheur - TÊTE EN FÊTE ${ }^{26}$ - l'anarchie même - VIVE L'ANARCHIE cibole ${ }^{27}$. Quant à la publicité pirate, à l'image de BUSINESS / MARDIFIFI ${ }^{28}$ (le nom d'une discothèque), elle cherche à multiplier ses interventions et pour cela utilise volontiers le pochoir, quand elle ne parasite pas les panneaux officiels ${ }^{29}$. Rien de très original d'ailleurs dans cette pratique... On n'a guère amélioré le scandale depuis la moustache imposée à la Joconde par les Futuristes et que, soit dit en passant, la véritable publicité n'a pas hésité à récupérer.

La déclaration d'intention, pour sa part, est toujours au bord du missionnariat: LA BOUFFE C'EST GRATUIT - / PIQUONS-LA!! ${ }^{30}$. Il s'agit, par un discours impératif, d'annoncer fermement ses couleurs puis de chercher à gagner des disciples. Et d'habitude, on ne rit pas! Il faut voir le ton: Soldats: descendez les chiens qui décident de vous envoyer au front ${ }^{31}$; VIVOTEZ FOREVER / GANG DE MOUTONS ${ }^{32}$; KILL EX.HIPPIES ! / YUPPIES ${ }^{33}$. À d'autres, heureusement moins sérieux, les interventions ludiques de toutes sortes qui vont du jeu de mots facile ou du dessin tout à fait gratuit ${ }^{34}$ aux trouvailles poétiques telles que VOS YEUX ESCLAVES / DE L'ORDINAIRE, d'une disposition plastique soignée et yeux grands ouverts reproduits pour plus d'efficacité ${ }^{35}$.

Autant de modes du graffito, autant de pactes graffiteur/graffitaire. Et autant de types de relations avec l'Institution. Informer, provoquer, insulter, amuser, ridiculiser, revendiquer suppose nécessairement que soient imaginés, fantasmés même, diverses attentes de la part des destinataires. Le graffito répond à cette nécessité qui sous-tend toute communication. Mais en y mettant une énergie spécifique; il se "positionne" marginalement (selon le vocabulaire de la pragmatique) par rapport aux règles admises par la société; il "braconne" celleci, et cette fois, c'est à de Certeau ${ }^{36}$ que nous nous référons; il prend le graffitage en otage en lui imposant une lecture que ce dernier n'a pas choisi de faire; il investit un espace interdit. Or, le geste d'écrire sur le mur de l'Autre est un geste d'agression. D'autant que cette "écriture " utilise des moyens violents - peinture le plus souvent en espace ouvert, que celle-ci soit appliquée au pinceau ou par bombe aérosol - qui le "maculent" longtemps.

Ibid., p. 21.

Ibid., p. 108.

Ibid., p. 85.

Ibid., p. 65.

Montréal graffiti bis, p. 49.

30 Montréal graffiti, p. 25.

31 Ibid., p. 41.

32 Ibid., p. 26.

33 Ibid., p. 96.

34 Ibid., p. 63 et 80.

35 Ibid., la page couverture.

36 Michel de Certeau, l'Invention du quotidien, t. I., Paris, Union générale d'éditions, 1980 $(10 / 18)$. 
Se servir du mur de l'Autre, c'est déjà abolir la neutralité à laquelle l'Autre a droit. Se servir du mur de l'Autre contre l'Autre, voilà l'arme par excellence. Aussi le graffito n'est-il jamais si fort que lorsqu'il s'exerce directement sur ou tout à côté de l'institution qu'il conteste. Nous en avons relevé plusieurs exemples à Montréal: dans la rue Saint-Jean-Baptiste qui mène au Palais de justice, les mots suivants, reliés, à quelques pieds de distance par une flèche et une graphie identique: JUSTICE $\rightarrow$ JAIL et qui trouve finalement leur conclusion dans le syntagme: LA GRANDE ILLUSSION [sic] ${ }^{37}$. Et d'ailleurs, tout près d'une caserne militaire, le graffito FOCK Les / militaires / VIRILS / et Violents ${ }^{38}$.

Bien que susceptibles de trouver un écho auprès de nombreux lecteurs, ces exemples, de toute évidence, témoignent d'une pulsion solitaire. Ils correspondent à une méthodologie personnelle, ils sont le fait d'un individu en mal de communiquer ses émotions et ses partis pris. Aussi leur pouvoir de mobilisation demeuret-il restreint malgré une performance textuelle certaine. Il en va tout autrement lorsque l'événement intéresse toute une communauté, la touche au cœur de ses préoccupations les plus fondamentales, la provoque, au point de déclencher des réactions en chaîne qui se multiplient dans l'espace et se précipitent dans le temps. D'individuel qu'il est par définition, le graffito passe alors au collectif; il perd son individualité thématique au profit $d u$ sujet à l'ordre du jour. Et il acquiert la force du manifeste. On a pu le constater lors des dernières élections chiliennes et encore tout récemment à l'occasion de la répression chinoise.

Des questions chaudes telles que l'avortement, le nucléaire, la pollution, entraînent volontiers, et quel que soit le pays, une abondance, voire une "épidémie" de graffiti. S'écrivent alors un peu partout en milieu urbain de grands textes passionnés, un peu simplistes souvent, parce que forcément réduits au slogan. Montréal n'est pas à l'abri de ces vagues de fond. La prolifération, à l'hiver 1989, d'une grande quantité d'inscriptions portant sur ou autour de la loi 101 en fait foi. Elles allaient du STOP maquillé en $101^{39}$ aux formules très angoissées comme [...] LAISSEZ-NOUS / EXISTER / OSTIE! / 101, 101 / = / PAIX / SOCIALE (voir photo p. 219), $178=$ COLONISÉ / QUÉBEC / FRANÇAIS $101^{40}$, etc. Et les opposants ne sont pas en reste qui, en plus d'associer systématiquement le symbole fasciste à la loi 101 , utilisent la parodie pour créer un effet boomerang: ENGLISH LIBERATION of QUEBEC / ELQ ${ }^{41}$. Si la portée du graffito dépend du sujet abordé, du lieu où il s'inscrit, d'une concentration dans le temps et dans l'espace et, on l'aura deviné, des moyens matériels qu'il emprunte, elle dépend beaucoup de la relation microtexte/macrotexte. Au point de s'en trouver souvent modifiée. Le graffito demeure-t-il seul, à l'exemple de cet AMNÉSIE ${ }^{42}$ repris cinq

37 Montréal graffiti, p. 114, pour la deuxième partie du graffito.

38 Ibid., p. 67.

39 Jeanne Demers, Josée Lambert et Line Mc Murray, Graffiti et Loi 101, Montréal, VLB éditeur, $1989, p .20$. Le phénomène a été si répandu que le service d'entretien n'a pu suffire à la tâche de remplacer les panneaux abîmés.

40 Ibid., p. 9 et 49.

41 Ibid., p. 13.

42 Montréal graffiti, p. 126. 
fois sur le mur latéral du métro Sherbrooke? Il s'enrichit du silence qui l'entoure, avec comme résultat un effet voisin de celui que produit le mantra. Le graffito entre-t-il au contraire dans un dia-multilogue? Sa signification initiale pourra subir un glissement plus ou moins important. Cela est arrivé au mot Tchernobyl, inscrit peu après le désastre écologique que l'on sait, et qu'un autre graffiteur a ensuite fait précéder de cet été on bronze mieux à, l'ironie ajoutant au tragique; qu'un autre encore a réinterprété en ajoutant, dans le rappel d'un titre de Boris Vian: J'IRAI ÉJACULER / SUR VOS BOMBES ${ }^{43}$. Preuve que le graffito est extrêmement vulnérable.

\section{Vulnérabilité du graffito et récupération par l'art}

Une fois lâché sur la place publique, le graffito échappe en effet à son auteur. Et les risques physiques, la pluie, la neige, le vent, les nettoyages périodiques qui l'attendent inévitablement sont, contrairement à ce que l'on pourrait penser, loin d'être les plus importants. Le graffito peut échapper à son auteur par simple déni de sens induit par une inscription subséquente, comme dans cette interprétation parodique du sigle F.A.R. pour LA Fraction Anti-Roger Vaincra / F.A.R. ${ }^{44} \mathrm{Ne}$ lui échappe-t-il pas jusqu'à un certain point dès qu'entraîné dans un dia ou multilogue? Le phénomène, plutôt rare en espace ouvert, est fréquent dans les lieux clos où il prend volontiers l'allure d'une véritable conversation. Un bon exemple: une plaque de bois dont les employés d'entretien d'une toilette $Q$ de cégep s'étaient servi pour couvrir un trou dans un mur et qui a été complètement recouverte de graffiti se répondant les uns aux autres autour de thèmes touchant l'amour et la sexualité ${ }^{45}$.

Le graffitaire, notons-le, se retrouve alors plus voyeur que spectateur. Lintimité des lieux y est, bien entendu, pour quelque chose. Mais il y a surtout le type même de macrotexte impliqué. Ne se déroule-t-il pas (ou ne paraît-il pas se dérouler?) comme en circuit fermé, entre graffiteurs, plaçant ainsi le lecteur non actif en position de graffitaire second? Lors de notre cueillette de graffiti extérieurs, nous avons fait la découverte étonnante d'un panneau sur lequel les prostituées du quartier se préviennent des dangers de leur pratique quotidienne. Un simple coup d'œil sur ce panneau et le lecteur se sent de trop: il est évident que les inscriptions qu'il y voit ne le concernent pas, qu'il s'agisse de switch blade, de Italien gros, de Knife ou des numéros de téléphone ou de plaque dimmatriculation qui y sont reproduits ${ }^{46}$.

Mais il y a plus: le graffito échappe à son graffiteur au fur et à mesure que le temps entame l'actualité du propos mis en scène. Cela va de soi... en principe; car certaines expressions comme cet HIROSHIMA ${ }^{47}$ maintenant disparu, mais dont la durée a été fort longue, jouent un rôle métaphorique qui dépasse l'événement. Parce que texte susceptible de devenir producteur de sens autres que celui initialement prévu, le graffito échappe encore plus fondamentalement à son

\footnotetext{
43 Ibid., p. 98.

44 Ibid., p. 90.

45 Montréal graffit bis, p. 104.

46 Montréal graffiti, p. 48.

47 Ibid., p. 23.
} 
auteur, d'autant plus lorsqu'il s'appuie sur l'intertextualité, comme dans ce bel exemple emprunté à Nelligan et réactualisé d'heureuse façon:

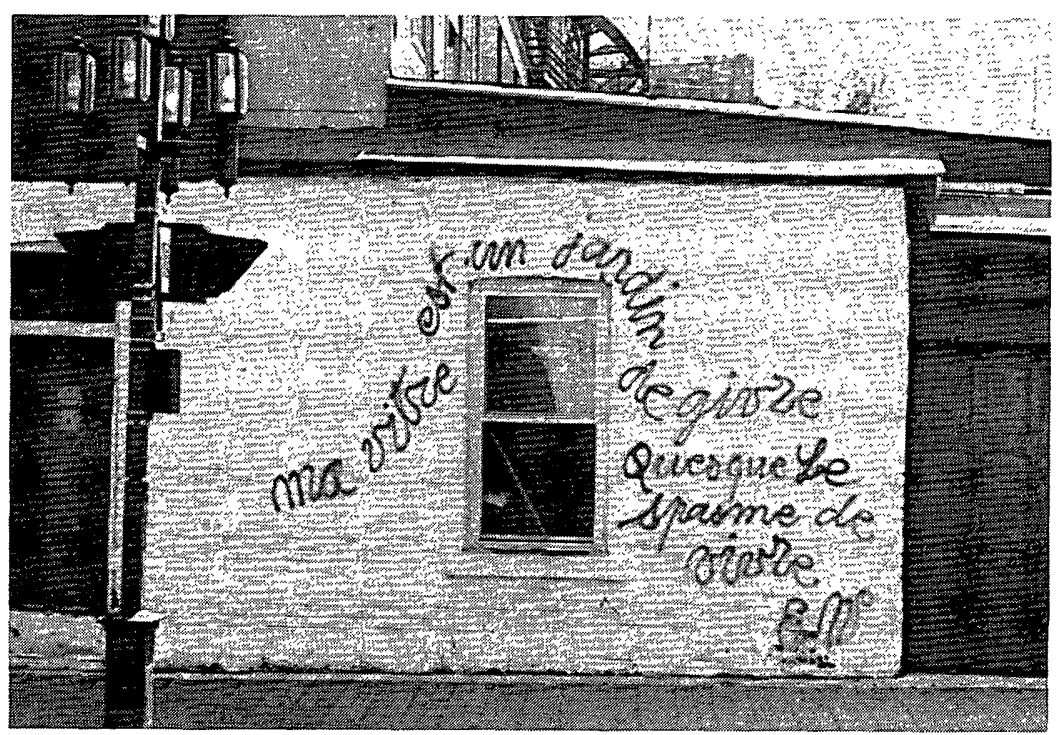

La vraie "perte» du graffito est particulièrement paradoxale. Elle est due d'une part à l'effet séduction-choc sans lequel il n'existerait pas, mais qui le place toujours sur les bords ambigus de l'œuvre, d'autre part à la lucidité dont il fait souvent preuve. Ainsi cette proclamation, une sorte de méta-graffito, qui se met elle-même en question - AFFICHEZ [sic] UN POĖME EST UN DÉLIT ${ }^{49}$ - ou cet autre qui associe le graffito à un mass média ${ }^{50}$.

Aussi est-ce très tôt la récupération ${ }^{51}$, puisqu'il faut l'appeler par son nom. Celle-ci prendra d'abord la forme subtile de graffiti signés par des artistes, comme à Montréal les "œuvres" de Zilon ou celles d'Horus-Ra ${ }^{52}$.

Ce sera ensuite la pression des galeries, comme à New York où l'on a été jusqu'à utiliser l'expression post graffiti ${ }^{53}$, quand ce ne sont pas les artistes euxmêmes qui, effectuant un retour à la rue, sinspirent du graffito - la toile de Zéro

48 Ibid., p. 130.

49 Ibid., p. 78.

50 Montréal graffiti bis, p. 17.

51 Il s'agit bien entendu de la récupération de la marginalité par le système. On pourrait examiner certaines formes du mur, de l'existence de certains objets, valves, par exemple (Montréal graffiti, p. 128), borne-fontaine (ibid., p. 80., parcometre (ibid., p. 63), etc. Mais cela est une autre question.

52 Ibid., p. 109.

53 Delphine Renard, « Graffiti-writers, Graffiti-artists „, Art Press, n 81, mai 1984, p. 9; reproduit dans Aérosol/Graffiti Art, Bruxelles, imprimerie Paulclean, $n^{\circ} 2 A$. 
Zoo, de son vrai nom François Pierre Bleau, intitulée Syntèze de grafiti ${ }^{54}$ - ou sollicitent la participation du public sous forme de graffiti. Nous pensons ici à la sculpture le Mur/Perspective d'infini-constats sociologiques de Tatiana Démidoff-Séguin ${ }^{55}$ qui reproduit à l'aide de ciment tondu vitrifié, de bois et de miroirs, une palissade recouverte de graffiti obtenus lors de l'exposition inaugurale.

Et il ne faudrait pas minimiser le rôle des reportages ni des quelques livresalbums européens ou américains déjà parus ${ }^{56}$. D'autant moins qu'ils se multiplient juste au moment où le phénomène paraît montrer des signes de déclin, à New York, par exemple...

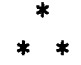

Traiter le graffito comme un phénomène social intéressant, le montrer en insistant sur son caractère manifestaire et sur ses qualités plastiques, n'est-ce pas admettre qu'il fait partie de l'Institution? N'est-ce pas l'intégrer au système? La question qui se pose alors: le graffito survivra-t-il à l'opération? Survivra-t-il à sa récupération par l'enseignement, par la critique? Survivra-t-il à la perversion de son utilisation en publicité commerciale, lorsqu'à rebours de son projet existentiel, il revendique, comme dans une annonce d'une compagnie de ciment ${ }^{57}$, rien de moins que le droit au profit: PROFITS / ... et pourquoi pas?

Question qui en appelle une autre: si le graffito devait disparaître, emporté par son propre succès ou du moins "contenu", comme avant les années 60 , dans les limites d'une pratique exceptionnelle, comment s'exprimera le nécessaire discours marginal quil suppose? L'existence d'une soupape est quelquefois heureuse...

54 Précis de zérozoïsme/art, filozofi, mœurs é mode de la cultur zérozoïste [...] précédé de «Lotobiografi romentic ... de Zéro $\mathrm{ZOO}$ *, peintre, idéologiste é créateur multiple de la reproduction intégrale de al'expo zérozoiste "/e tout formant un roman thriller très réel, Montréal, éd. Roxane Turcotte, 1986, p. 100.

55 Cf. le catalogue de ses œuvres, 1986, éd. D.S. Lorraine, p. 26. Cette œuvre a mérité le prix 1984 du Conseil de la sculpture du Québec.

56 Pino Marchi, Italia Spray/Storia del Ultima Italia Scritta sui muri, Firenze, Vallecchi, 1977,160 p. The Faith of Graffiti, documented by Mervyn Kuriansky et John Naar, text by Norman Mailer, New York, Praegrer Publ., 1974 [n.p.]. Les Murs murmurent, ils crient, ils chantent..., collectif, Paris, éd. du Centre Georges-Pompidou, 1982, 56 p.

57 Parue dans l'Actualité, mai 1982, p. 46. Il s'agit d'une publicité de Ciments Canada Lafarge ltée. 
